\title{
VEHICLE RECOGNITION USING VIBE AND SVM
}

\author{
Jinlin Liu, Qiang Chen and Chen Zhang \\ College of Electronic and Electrical Engineering, Shanghai University of Engineering \\ Science, Shanghai 201620,China.
}

\begin{abstract}
Video surveillance is becoming more and more important forsocial security, law enforcement, social order,military, and other social problems. In order to manage parking information effectively, this vehicle detection method is presented. In general, motion detection plays an important role in video surveillance systems. In this paper, firstly this system uses ViBe method to extract the foreground object, then extracts HOG features on the performance of the ROI of images. At last this paper presents Support vector machine for vehicle recognition. The results of this test show that, the recognition rate of vehicle's model in this recognition system is up the industrial application standard.
\end{abstract}

\section{KEYWORDS}

ViBe, SVM, vehicle recognition, $H O G$

\section{INTRODUCTION}

With the development of the city, more and more people have their own vehicles. Meanwhile, the speed of parking lot construction cannot compare with the rate of car growth. So there was a contradiction between people and between the supply and demand of parking. In order to build smart city and facilitate people's lives, we have to solve this problem[1].

Now in many cities have been building many parking lots which have a very high level of automation, and can remind divers how many free parking locations when they entered the parking lots. Even some of the higher-end parking lot offers Apps, and divers can book theirs parking space online. But for those who do not plan well site, monitoring of vehicles are very loose, or in a state of lack of supervision.[2] There is no doubt that this situation will give some unpleasant feelings. Therefore, in order to better build smart cities, planning and management of outdoor parking lots is necessary.

This paper introduces how to tell the moving vehicles from parking lot surveillance video. Using ViBe background modeling method for moving target detection to identify moving objects. This

DOI: $10.5121 /$ cseij.2016.6402 
Computer Science \& Engineering: An International Journal (CSEIJ), Vol.6, No.4, August 2016

system uses SVM method determines whether the object is a vehicle, and judge the state of motion of the vehicle, and then updates the information of parking spaces.

\section{BACKGROUND SUBTRACTION ALGORITHMS}

Locating moving objects in a video sequence is the first step of many computer vision applications.Many background subtraction techniques have been proposed with as many models and segmentation strategies, and several surveys are devoted to this topic.This system uses ViBe method detecting moving foreground objects

\subsection{Brief introduction of VIBE}

Background differencing is the commonly used method for the detection of moving objects in the static background, and the ViBe algorithm is the main modeling approach. our proposed technique stores, for each pixel, a set of values taken in the past at the same location or in the neighborhood. It then compares this setting for the current pixel value to determine if the pixel belongs to the background, and adapts to the model of a random selection of values instead of from the background model. This method is different from those based on the oldest values of the classical belief should be replaced first.

Finally, if the pixel is one part of the background, then its value will be propagated into the background model and update the model after judgement.

ViBe algorithm using neighboring pixels in the color space determine the current pixel is the foreground pixels or background pixels, and it establishes a background pixel samples for each pixel value space, and save the pixel and its neighboring pixels in the sample space recent background pixel values. The current pixel-by-pixel with the pixel values of the background models with the most similar comparison, value as long as the current pixel-by-pixel with the

background part of the pixel values in the model, determine the background pixels pixel, pixel background model can be expressed as

$$
M(x)=\left\{v_{1}, v_{2}, \ldots, v_{N}\right\}
$$

In this paper, we denote by $\mathrm{v}(\mathrm{x})$ the value in a given Euclidean color space taken by the pixel located at $\mathrm{x}$ in the image, and by $v_{\mathrm{i}}$ a background sample value with an index i. Each background pixel $\mathrm{x}$ is modeled by a collection of $\mathrm{N}$ background sample values.[3]

VIBE initialization algorithms use the first frame image background model for every sample value of the background pixels in the sample space from the pixel and its neighboring pixels, selects a random pixel values to initialize.Each pixel has the same probability of being chosen. The model can be expressed as 
$M^{0}(x)=\left\{v^{0}\left(y \mid y \in N_{G}(x)\right)\right\}$

$\mathrm{y}$ is one pixel selected in the neighborhood of $\mathrm{x}$ randomly, and $N_{G}(x)$ is the collection of neighborhoods. $\mathrm{v}(\mathrm{y})$ pixel values may appear more than once in the background model.

$\mathrm{ViBe}$ algorithm starts to detect foreground objects from second frame. In order to classify a pixel value $\mathrm{v}(\mathrm{x})$ on the basis of its corresponding model $\mathrm{M}(\mathrm{x})$, we compare it to the closest values within the set of samples by defining a sphere $S_{R}(v(x))$ of radius R centered on $\mathrm{v}(\mathrm{x})$ [3].If thegiven threshold \#min is less than thecount of the set junction of sphere and the collection of model samples $\mathrm{M}(\mathrm{x})$, the pixel value $\mathrm{v}(\mathrm{x})$ will be classified as background. More formally, we compare $\#$ min to

$$
\sharp\left\{S_{R}(v(x)) \cap\left\{v_{1}, v_{2}, \ldots, v_{N}\right\}\right\}
$$

If $\mathrm{M}(\mathrm{x})$ is larger than $\sharp \mathrm{min}$, the pixel $\mathrm{x}$ is background pixel, otherwise $\mathrm{x}$ is foreground pixel [4]. We can learn that directly from this figure.

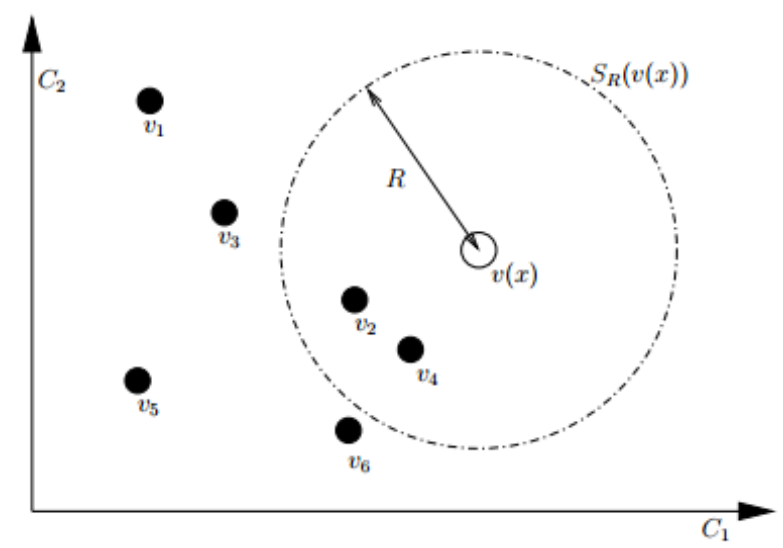

Fig.1. Comparison of a pixel value with a set of samples in a two dimensional Euclidean color space (C1,C2). To classify $\mathrm{v}(\mathrm{x})$, we count the number of samples of $\mathrm{M}(\mathrm{x})$ interesting the sphere of radius $\mathrm{R}$ centered on $\mathrm{v}(\mathrm{x})$.

\subsection{Experimental Results}

For the experiments, the proposed method was implemented using C++ with OpenCV library. In this experiment, we set the radius $\mathrm{R}$ of the sphere used to compare a new pixel value to pixel samples, the time subsampling factor $\Phi$, the number $\mathrm{N}$ of samples stored in each pixel model, and the number $\#$ min of close pixel samples needed to classify a new pixel value as background. In our experience, we set radius $R=20$, and time subsampling factor $\Phi=16, \quad N=20$, and set $\#$ min=20. 
Our dataset is made of videos from parking lot, we pick a pieceof video for experiment to test the effect of ViBe algorithm. In order to highlight the prospects for detection of objects and have a good performance, we add the code of merge window.



(a)

(b)

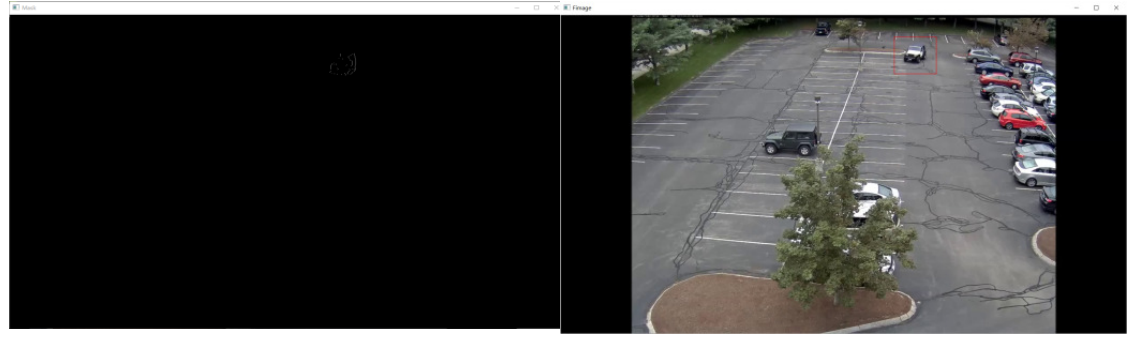

(c)

(d)

Fig.2. Input video is (a), after the process of foreground objects detection is (b),After morphological processing and edge processing is (c). Locating moving objects region in the picuture(d)

\section{VEHICLES RECOGNITION}

When after receiving the moving image, the next we are going to determine whether objects are vehicles. There are many ways to classify, such as logistic regression analysis, kernel logistic regression, Support Vector Machine (SVM). Support vector machine is a new and rapidly developing methods of machine learning and classification, are widely used in many types of classification and pattern recognition.

\subsection{Briefintroduction of SVM}

SVMs are primarily two-class classifiers that have been proved thatit is an effective and more robust method to handle linear or non-linear decision boundaries [5] [6] . SVM will find the hyperplanebya set of points, which belong to either of two classes. This hyperplanehas the largest distance to each class, and the largest possible fraction of points of the same class on the same 
Computer Science \& Engineering: An International Journal (CSEIJ), Vol.6, No.4, August 2016

side.This is equivalent to the implementation of structural risk minimization to achieve good generalization [5] [6]. Assuming 1 examples from two classes.

In this section, We suppose we have a set $\mathrm{S}$ which has $\mathrm{N}$ points of training samples, $x_{i} \in \mathbb{R}^{w}$ withi $=$ $1,2, \ldots, \mathrm{N}$. Each point $x_{i}$ belong to one of two classes and is given a label $y_{i} \in\{-1,1\}$.Our purposeis to build a hyperplane equationthat divides $S$ into two classes correctly, and maximize the distance between the two classes and the hyperplane [6]. A hyperplane can be described as the equationin the feature space.

$$
<w, x>+b=0(4)
$$

where $w \in \mathbb{R}^{w}$ and $\mathrm{b}$ is a scalar. When the training samples are linearly separable, Optimal hyperplane of SVM, which can separate the two classes, no training mistakes and maximize the minimum distance from the sample to the hyperplane.

\subsection{Histograms of Oriented Gradients (HOG)}

Histogram of oriented gradients (HOG) feature is a descriptor for computer vision and image processing todetect object. Through calculations and statistical characteristics of gradient orientation histogram of image local area to form. Hog combination of SVM classifiers have been widely used in image recognition, especially in the detection of pedestrians has a great performance.In practice, the orientation range is divided into $\beta$ bins, and each pixel in the region votes for its corresponding bin.

\subsection{Experimental Results}

Framework of the vehicle recognition as shown in the figure: including learning and testing phases. During the learning phase, mainly is the integration of collected data, get these features, then entered into a learning classifier, to generate the target classifier. After entering the testing phase, you need to use the classification obtained in the previous step, by comparing the scan window and then click the input image, is judged to be identified in accordance with the classifier of the small area [7][8]. 
Computer Science \& Engineering: An International Journal (CSEIJ), Vol.6, No.4, August 2016

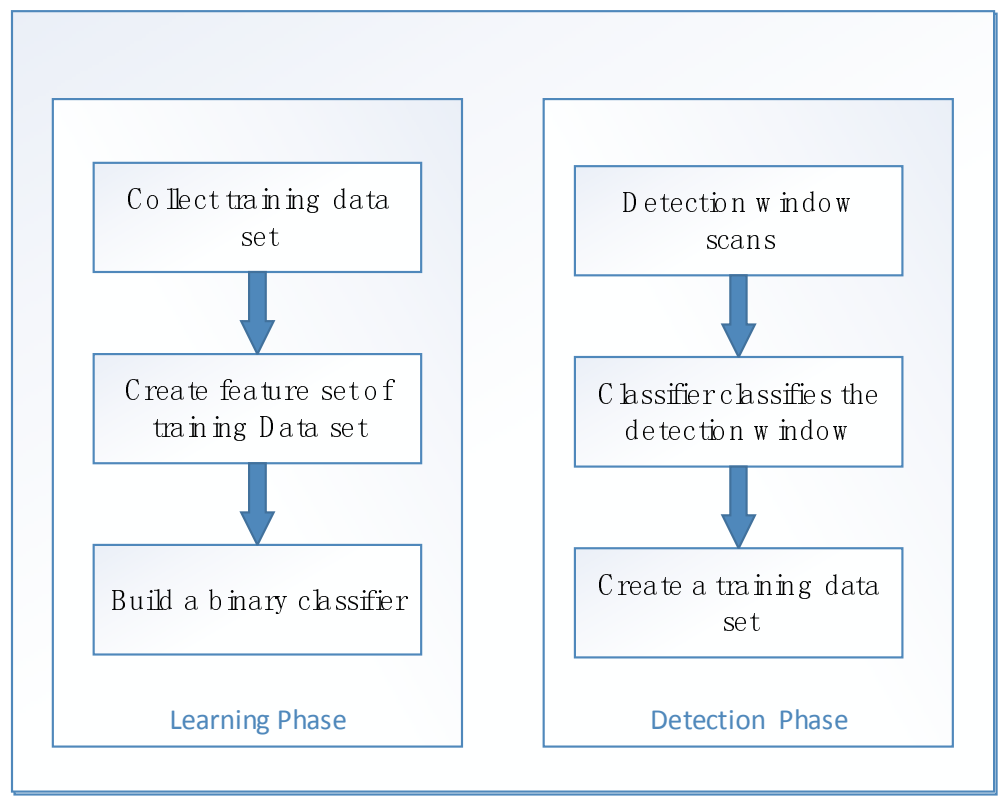

Fig.3 Flow chart of SVM

In our experiment, we collect training data from ImageNet, we download the pictures, and resize them $32 * 32$, so the feature dimensions of each image is 1764 .

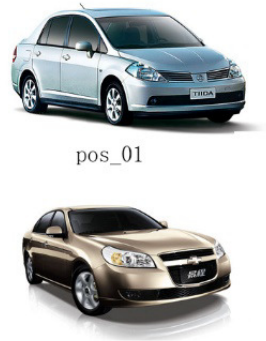

pos_05

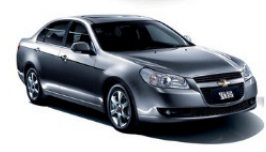

pos_09

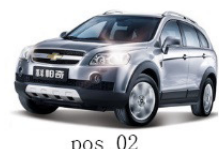

$$
\text { pos_02 }
$$

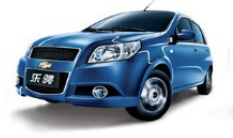

pos_06

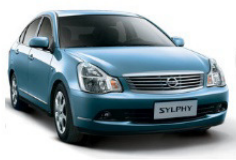

pos_10
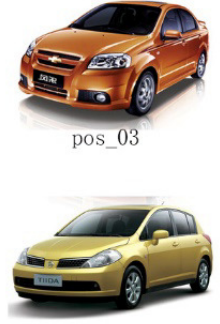

pos_07

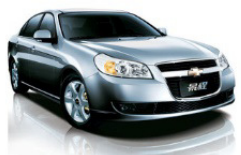

pos_11


pos_08

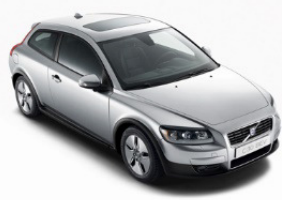

pos_12

Fig.4 positivesample images

After the system completes the learning phase, the system will judge the foreground objects. Flow 
chart is as follows.

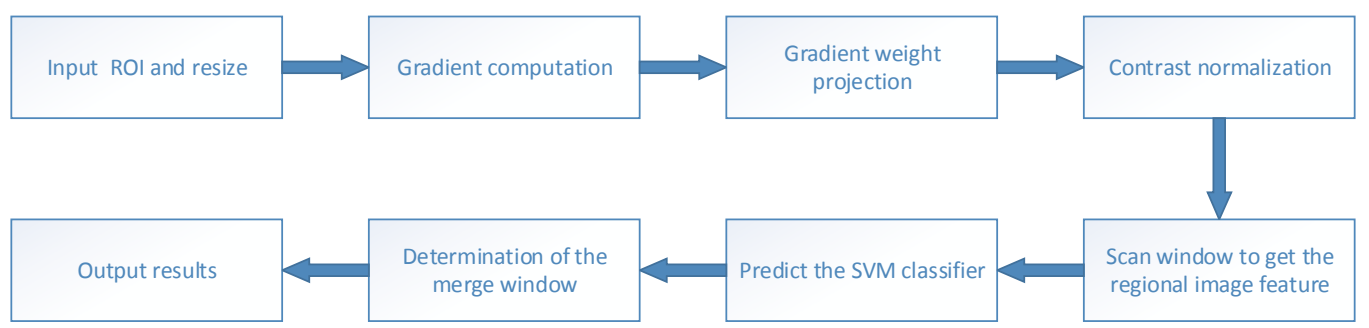

Fig.5 detction process flow chart

In this section, the HOG feature of foreground objects will be sent to SVM. Every window will be tested and predicted by SVM. The SVM will output "-1" or "1". The"1" presents the vehicle,"-1" presents non-vehicle. This information will show in a new window.



Fig.6 After passing vehicle recognition, we remove the vehicle pictures and displays


Fig.7 This design can detect more than 2 vehicles at the same time. 
Computer Science \& Engineering: An International Journal (CSEIJ), Vol.6, No.4, August 2016

\section{CONCLUSION}

We have considered the problem of vehicle detection from video surveillance of parking lots. The HOG can describe vehicle information, handle within-class variations, and global illumination insensitive to changes in.

The first part of the study is devoted to the analysis of the performance of ViBe for foreground objects detection. SVM based on HOG features is shown to achieve the good results. However, the outcome of the study shows that, results with high accuracy, but the efficiency problem happens in certain situations. The outcome of experiments reveals that SVM can work well in normal situation, but in some extreme situationssuch as tInclement weather, the effectofrecognition of a little bad.In order to enhance the robustness and accuracy of the system, we plan to integrate other features (for example, LBP feature), and use optical flow method to optimizethe moving object detection and background modelling.

\section{REFERENCES}

[1] S. Suryanto, D.-H. Kim, H.-K. Kim, and S.-J. Ko, "Spatial colorhistogram based center voting method for subsequent objecttracking and segmentation," Image and Vision Computing, vol.29, no. 12, pp. 850-860, 2011.

[2] J. Hwang, K. Huh, and D. Lee, "Vision-based vehicle detection and tracking algorithm design," Optical Engineering, vol. 48, no.12, Article ID 127201, 2009.

[3] O. Barnich and M. Van Droogenbroeck, "ViBe: a powerful random technique to estimate the background in video sequences," in Int. Conf.on Acoustics, Speech and Signal Processing (ICASSP), pp. 945-948,April 2009.

[4] C. Burges, "Tutorial on support vector machines for pattern recognition," Data Mining and Knowledge Discovery, vol. 2, no. 2, pp. 955-974, 1998.

[5] L.-W. Tsai, J.-W. Hsieh, and K.-C. Fan, "Vehicle detection using normalized color and edge map," IEEE Transactions on Image Processing, vol. 16, no. 3, pp. 850-864, 2007.

[6] V. Vapnik, The Nature of Statistical Learning Theory. Springer Verlag, 1995.

[7] M. Van Droogenbroeck and O. Barnich, "Visual background extractor."World Intellectual Property Organization, WO 2009/007198, 36 pages, January 2009.

[8] Joshua Gleason, Ara V. Nefian, Xavier Bouyssounousse, Terry Fong and George Bebis 2011

[9] G'erardBiau, Luc Devroye, and G'abor Lugosi. Consistency of random forests and other averaging classifiers. J. Mach. Learn. Res., 9:2015-2033, 2008.

[10] C. N. Anagnostopoulos, I. Giannoukos, T. Alexandropoulos, A. Psyllos, V. Loumos, and E. Kayafas, "Integrated vehicle recognition and inspection system to improve security in restricted access areas," in Intelligent Transportation Systems (ITSC)", 2010 13th International IEEE Conference on, 2010, pp. 1893-898. [3] L. Bottou, C. Cortes, J. 
Computer Science \& Engineering: An International Journal (CSEIJ), Vol.6, No.4, August 2016

\section{AUTHORS}

Jinlin Liu is currently studying in Mechanical and Electronic Engineering fromShanghai University of Engineering Science, China, where he is working towards theMaster degree. His current research interests include image processing, machine learning

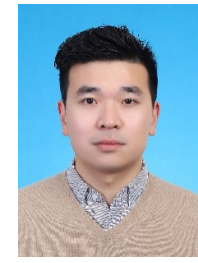

